\title{
Research Article \\ On Fixed Points of Maximalizing Mappings in Posets
}

\author{
S. Heikkilä \\ Department of Mathematical Sciences, University of Oulu, P.O. Box 3000, 90014 Oulu, Finland
}

Correspondence should be addressed to S. Heikkilä, sheikki@cc.oulu.fi

Received 7 October 2009; Accepted 16 November 2009

Academic Editor: Mohamed A. Khamsi

Copyright $@ 2010$ S. Heikkilä. This is an open access article distributed under the Creative Commons Attribution License, which permits unrestricted use, distribution, and reproduction in any medium, provided the original work is properly cited.

We use chain methods to prove fixed point results for maximalizing mappings in posets. Concrete examples are also presented.

\section{Introduction}

According to Bourbaki's fixed point theorem (cf. $[1,2]$ ) a mapping $G$ from a partially ordered set $X=(X, \leq)$ into itself has a fixed point if $G$ is extensive, that is, $x \leq G(x)$ for all $x \in X$, and if every nonempty chain of $X$ has the supremum in $X$. In [3, Theorem 3] the existence of a fixed point is proved for a mapping $G: X \rightarrow X$ which is ascending, that is, $G(x) \leq y$ implies $G(x) \leq G(y)$. It is easy to verify that every extensive mapping is ascending. In [4] the existence of a fixed point of $G$ is proved if $a \leq G(a)$ for some $a \in X$, and if $G$ is semi-increasing upward, that is, $G(x) \leq G(y)$ whenever $x \leq y$ and $G(x) \leq y$. This property holds, for instance, if $G$ is ascending or increasing, that is, $G(x) \leq G(y)$ whenever $x \leq y$.

In this paper we prove further generalizations to Bourbaki's fixed point theorem by assuming that a mapping $G: X \rightarrow X$ is maximalizing, that is, $G(x)$ is a maximal element of $\{x, G(x)\}$ for all $x \in X$. Concrete examples of maximalizing mappings $G$ which have or do not have fixed points are presented. Chain methods introduced in $[5,6]$ are used in the proofs. These methods are also compared with three other chain methods.

\section{Preliminaries}

A nonempty set $X$, equipped with a reflexive, antisymmetric, and transitive relation $\leq$ in $X \times X$, is called a partially ordered set (poset). An element $b$ of a poset $X$ is called an upper 
bound of a subset $A$ of $X$ if $x \leq b$ for each $x \in A$. If $b \in A$, we say that $b$ is the greatest element of $A$, and denote $b=\max A$. A lower bound of $A$ and the least element, $\min A$, of $A$ are defined similarly, replacing $x \leq b$ above by $b \leq x$. If the set of all upper bounds of $A$ has the least element, we call it the supremum of $A$ and denote it by sup $A$. We say that $y$ is a maximal element of $A$ if $y \in A$, and if $z \in A$ and $y \leq z$ imply that $y=z$. The infimum of $A$, inf $A$, and a minimal element of $A$ are defined similarly. A subset $W$ of $X$ is called a chain if $x \leq y$ or $y \leq x$ for all $x, y \in W$. We say that $W$ is well ordered if nonempty subsets of $W$ have least elements. Every well-ordered set is a chain.

Let $X$ be a nonempty poset. A basis to our considerations is the following chain method (cf. [6, Lemma 2]).

Lemma 2.1. Given $G: X \rightarrow X$ and $a \in X$, there exists a unique well-ordered chain $C$ in $X$, called $a$ w-o chain of aG-iterations, satisfying

$$
x \in C \quad \text { iff } x=\sup \left\{a, G\left[C^{<x}\right]\right\} \text {, where } C^{<x}=\{y \in C: y<x\} \text {. }
$$

If $x_{*}=\sup \{a, G[C]\}$ exists in $X$, then $x_{*}=\max C$, and $G\left(x_{*}\right) \leq x_{*}$.

The following result helps to analyze the w-o chain of $a G$-iterations.

Lemma 2.2. Let $A$ and $B$ be nonempty subsets of $X$. If $\sup A$ and $\sup B$ exist, then the equation

$$
\sup (A \cup B)=\sup \{\sup A, \sup B\}
$$

is valid whenever either of its sides is defined.

Proof. The sets $A \cup B$ and $\{\sup A, \sup B\}$ have same upper bounds, which implies the assertion.

A subset $W$ of a chain $C$ is called an initial segment of $C$ if $x \in W, y \in C$, and $y<x$ imply $y \in W$. If $W$ is well ordered, then every element $x$ of $W$ which is not the possible maximum of $W$ has a successor: $S x=\min \{y \in W: x<y\}$, in $W$. The next result gives a characterization of elements of the w-o chain of $a \mathrm{G}$-iterations.

Lemma 2.3. Given $G: X \rightarrow X$ and $a \in X$, let $C$ be the w-o chain of aG-iterations. Then the elements of $\mathrm{C}$ have the following properties.

(a) $\min C=a$.

(b) An element $x$ of $C$ has a successor in $C$ if and only if $\sup \{x, G(x)\}$ exists and $x<$ $\sup \{x, G(x)\}$, and then $S x=\sup \{x, G(x)\}$.

(c) If $W$ is an initial segment of $C$ and $y=\sup W$ exists, then $y \in C$.

(d) If $a<y \in C$ and $y$ is not a successor, then $y=\sup C^{<y}$.

(e) If $y=\sup C$ exists, then $y=\max C$. 
Proof. (a) $\min C=\sup \left\{a, G\left[C^{<\min C}\right]\right\}=\sup \{a, G[\emptyset]\}=\sup \{a, \emptyset\}=a$.

(b) Assume first that $x \in C$, and that $S x$ exists in C. Applying (2.1), Lemma 2.2, and the definition of $S x$ we obtain

$$
S x=\sup \left\{a, G\left[C^{<S x}\right]\right\}=\sup \left\{a, G\left[C^{<x}\right] \cup\{G(x)\}\right\}=\sup \{x, G(x)\}
$$

Moreover, $x<S x$, by definition, whence $x<\sup \{x, G(x)\}$.

Assume next that $x \in C$, that $y=\sup \{x, G(x)\}$ exists, and that $x<\sup \{x, G(x)\}$. The previous proof implies the following

(i) There is no element $w \in C$ which satisfies $x<w<\sup \{x, G(x)\}$.

Then $\{z \in C: z \leq x\}=C^{<y}$, so that

$$
\begin{aligned}
x<\sup \{x, G(x)\} & =\sup \left\{\sup \left\{a, G\left[C^{<x}\right]\right\}, G(x)\right\} \\
& =\sup \left\{\{a\} \cup G\left[C^{<x}\right] \cup\{G(x)\}\right\} \\
& =\sup \{a, G[\{z \in C: z \leq x\}]\} \\
& =\sup \left\{a, G\left[C^{<y}\right]\right\} .
\end{aligned}
$$

Thus $y=\sup \{x, G(x)\} \in C$ by (2.1). This result and (i) imply that $y=\sup \{x, G(x)\}=$ $\min \{z \in C: x<z\}=S x$.

(c) Assume that $W$ is an initial segment of $C$, and that $y=\sup W$ exists. If there is $x \in W$ such that $S x \notin W$, then $x=\max W=y$, so that $y \in C$. Assume next that every element $x$ of $W$ has the successor $S x$ in $W$. Since $S x=\sup \{x, G(x)\}$ by (b), then $G(x) \leq S x<y$. This holds for all $x \in W$. Since $a=\min C=\min W<y$, then $y$ is an upper bound of $\{a\} \cup G[W]$. If $z$ is an upper bound of $\{a\} \cup G[W]$, then $x=\sup \left\{a, G\left[C^{<x}\right]\right\}=\sup \left\{a, G\left[W^{<x}\right]\right\} \leq z$ for every $x \in W$. Thus $z$ is an upper bound of $W$, whence $y=\sup W \leq z$. But then $y=\sup \{a, G[W]\}=$ $\sup \left\{a, G\left[C^{<y}\right]\right\}$, so that $y \in C$ by (2.1).

(d) Assume that $a<y \in C$, and that $y$ is not a successor of any element of $C$. Obviously, $y$ is an upper bound of $C^{<y}$. Let $z$ be an upper bound of $C^{<y}$. If $x \in C^{<y}$, then also $S x \in C^{<y}$ since $y$ is not a successor. Because $S x=\sup \{x, G(x)\}$ by (b), then $G(x) \leq S x \in C^{<y}$. This holds for every $x \in C^{<y}$. Since also $a \in C^{<y}$, then $z$ is an upper bound of $\{a\} \cup G\left[C^{<y}\right]$. Thus $y=\sup \left\{a, G\left[C^{<y}\right]\right\} \leq z$. This holds for every upper bound $z$ of $C^{<y}$, whence $y=\sup C^{<y}$.

(e) If $y=\sup C$ exists, then $y \in C$ by (c) when $W=C$, whence $y=\max C$.

In the case when $a \leq G(a)$ we obtain the following result (cf. [7, Proposition 1]).

Lemma 2.4. Given $G: X \rightarrow X$ and $a \in X$, there exists a unique well-ordered chain $C(a)$ in $X$, calleda w-o chain of G-iterations of a, satisfying

$$
a=\min C, \quad x \in C \backslash\{a\} \text { iff } x=\sup G\left[C^{<x}\right] .
$$

If $a \leq G(a)$, and if $x_{*}=\sup G[C(a)]$ exists, then $a \leq x_{*}=\max C(a)$, and $G\left(x_{*}\right) \leq x_{*}$. 
Lemma 2.4 is in fact a special case of Lemma 2.1, since the assumption $a \leq G(a)$ implies that $C(a)$ equals to the $\mathrm{w}$-o chain of $a \mathrm{G}$-iterations. As for the use of $C(a)$ in fixed point theory and in the theory of discontinuous differential and integral equations, see, for example, $[8,9]$ and the references therein.

\section{Main Results}

Let $X=(X, \leq)$ be a nonempty poset. As an application of Lemma 2.1 we will prove our first existence result.

Theorem 3.1. A mapping $G: X \rightarrow X$ has a fixed point if $G$ is maximalizing, that is, $G(x)$ is a maximal element of $\{x, G(x)\}$ for all $x \in X$, and if $x_{*}=\sup \{a, G[C]\}$ exists in $X$ for some $a \in X$ where $C$ is the w-o chain of a G-iterations.

Proof. If $C$ is the w-o chain of $a G$-iterations, and if $x_{*}=\sup \{a, G[C]\}$ exists in $X$, then $x_{*}=$ max $C$ and $G\left(x_{*}\right) \leq x_{*}$ by Lemma 2.1. Since $G$ is maximalizing, then $G\left(x_{*}\right)=x_{*}$, that is, $x_{*}$ is a fixed point of $G$.

The next result is a consequence of Theorem 3.1. and Lemma 2.3(e).

Proposition 3.2. Assume that $G: X \rightarrow X$ is maximalizing. Given $a \in X$, let $C$ be the w-o chain of $a G$-iterations. If $z=\sup C$ exists, it is a fixed point of $G$ if and only if $x_{*}=\sup \{z, G(z)\}$ exists.

Proof. Assume that $z=\sup C$ exists. It follows from Lemma 2.3(e) that $z=\max C$. If $z$ is a fixed point of $G$, that is, $z=G(z)$, then $x_{*}=\sup \{z, G(z)\}=z$, and $x_{*}=G\left(x_{*}\right)$. obtain

Assume conversely that $x_{*}=\sup \{z, G(z)\}$ exist. Applying (2.1) and Lemma 2.2 we

$$
\begin{aligned}
x_{*} & =\sup \{z, G(z)\}=\sup \left\{\sup \left\{a, G\left[C^{<z}\right]\right\}, \sup \{G(z)\}\right\} \\
& =\sup \left\{\{a\} \cup G\left[C^{<z}\right] \cup\{G(z)\}\right\}=\sup \{a, G[C]\} .
\end{aligned}
$$

Thus, by Theorem 3.1, $x_{*}=\max C=z$ is a fixed point of $G$.

As a consequence of Proposition 3.2 we obtain the following result.

Corollary 3.3. If nonempty chains of $X$ have supremums, if $G: X \rightarrow X$ is maximalizing, and if $\sup \{x, G(x)\}$ exists for all $x \in X$, then for each $a \in X$ the maximum of the $w$-o chain of a $G$-iterations exists and is a fixed point of $G$.

Proof. Let $C$ be the w-o chain of $a \mathrm{G}$-iterations. The given hypotheses imply that both $z=$ $\sup C$ and $x_{*}=\sup \{z, G(z)\}$ exist. Thus the hypotheses of Proposition 3.2 are valid.

The results of Lemma 2.3 are valid also when $C$ is replaced by the w-o chain $C(a)$ of $G$-iterations of $a$. As a consequence of these results and Lemma 2.4 we obtain the following generalizations to Bourbaki's fixed point theorem. 
Theorem 3.4. Assume that $G: X \rightarrow X$ is maximalizing, and that $a \leq G(a)$ for some $a \in X$, and let $C(a)$ be the w-o chain of G-iterations of $a$.

(a) If $x_{*}=\sup G[C(a)]$ exists, then $x_{*}=\max C(a)$, and $x_{*}$ is a fixed point of $G$.

(b) If $z=\sup C(a)$ exists, it is a fixed point of $G$ if and only if $x_{*}=\sup \{z, G(z)\}$ exists.

(c) If nonempty chains of $X$ have supremums, and if $\sup \{x, G(x)\}$ exists for all $x \in X$, then $x_{*}=\max C(a)$ exists, and $x_{*}$ is a fixed point of $\mathrm{G}$.

The previous results have obvious duals, which imply the following results.

Theorem 3.5. A mapping $G: X \rightarrow X$ has a fixed point if $G$ is minimalizing, that is, $G(x)$ is a minimal element of $\{x, G(x)\}$ for all $x \in X$, and if $\inf \{a, G[W]\}$ exists in $X$ for some $a \in X$ whenever $W$ is a nonempty chain in $X$.

Theorem 3.6. A minimalizing mapping $G: X \rightarrow X$ has a fixed point if inf $G[W]$ exists whenever $W$ is a nonempty chain in $X$, and if $G(a) \leq a$ for some $a \in X$.

Proposition 3.7. A minimalizing mapping $G: X \rightarrow X$ has a fixed point if every nonempty chain $X$ has the infimum in $X$, and if $\inf \{x, G(x)\}$ exists for all $x \in X$.

Remark 3.8. The hypothesis that $G: X \rightarrow X$ is maximalizing can be weakened in Theorems 3.1 and 3.4 and in Proposition 3.2 to the form: $G \mid\left\{x_{*}\right\}$ is maximalizing, that is, $G\left(x_{*}\right)$ is a maximal element of $\left\{x_{*}, G\left(x_{*}\right)\right\}$.

\section{Examples and Remarks}

We will first present an example of a maximalizing mapping whose fixed point is obtained as the maximum of the w-o chain of $a G$-iterations.

Example 4.1. Let $X$ be a closed disc $X=\left\{(u, v) \in \mathbb{R}^{2}: u^{2}+v^{2} \leq 2\right\}$, ordered coordinate-wise. Let $[u]$ denote the greatest integer $\leq u$ when $u \in \mathbb{R}$. Define a function $G: X \rightarrow \mathbb{R}^{2}$ by

$$
G(u, v)=\left(\min \{1,1-[u]+[v]\}, \frac{1}{2}\left([u]+v^{2}\right)\right), \quad(u, v) \in X .
$$

It is easy to verify that $G[X] \subset X$, and that $G$ is maximalizing. To find a fixed point of $G$, choose $a=(1,0)$. It follows from Lemma 2.3(b) that the first elements of the w-o chain of $a \mathrm{G}$-iterations are successive approximations

$$
x_{0}=a, \quad x_{n+1}=S x_{n}=\sup \left\{x_{n}, G\left(x_{n}\right)\right\}, \quad n=0,1, \ldots,
$$

as long as $S x_{n}$ is defined. Denoting $x_{n}=\left(u_{n}, v_{n}\right)$, these successive approximations can be rewritten in the form

$$
\begin{aligned}
& u_{0}=1, \quad u_{n+1}=\max \left\{u_{n}, \min \left\{1,1-\left[u_{n}\right]+\left[v_{n}\right]\right\}\right\}, \\
& v_{0}=0, \quad v_{n+1}=\max \left\{v_{n}, \frac{1}{2}\left(\left[u_{n}\right]+v_{n}^{2}\right)\right\}, \quad n=0,1, \ldots,
\end{aligned}
$$


as long as $u_{n} \leq u_{n+1}$ and $v_{n} \leq v_{n+1}$, and at least one of these inequalities is strict. Elementary calculations show that $u_{n}=1$, for every $n \in \mathbb{N}_{0}$. Thus (4.3) can be rewritten as

$$
u_{n}=1, \quad v_{0}=0, \quad v_{n+1}=\max \left\{v_{n}, \frac{1}{2}\left(1+v_{n}^{2}\right)\right\}, \quad n=0,1, \ldots
$$

Since the function $g(v)=(1 / 2)\left(1+v^{2}\right)$ is increasing $\mathbb{R}_{+}$, then $v_{n}<g\left(v_{n}\right)$ for every $n=0,1, \ldots$. Thus (4.4) can be reduced to the form

$$
u_{n}=1, \quad v_{0}=0, \quad v_{n+1}=g\left(v_{n}\right)=\frac{1}{2}\left(1+v_{n}^{2}\right), \quad n=0,1, \ldots
$$

The sequence $\left(g\left(v_{n}\right)\right)_{n=0}^{\infty}$ is strictly increasing, whence also $\left(v_{n}\right)_{n=0}^{\infty}$ is strictly increasing by (4.5). Thus the set $W=\left\{\left(1, g\left(v_{n}\right)\right)\right\}_{n \in \mathbb{N}_{0}}$ is an initial segment of $C$. Moreover, $v_{0}=0<1$, and if $0 \leq v_{n}<1$, then $0<g\left(v_{n}\right)<1$. Since $\left(g\left(v_{n}\right)\right)_{n=0}^{\infty}$ is bounded above by 1 , then $v_{*}=$ $\lim _{n} g\left(v_{n}\right)$ exists, and $0<v_{*} \leq 1$. Thus $\left(1, v_{*}\right)=\sup W$, and it belongs to $X$, whence $\left(1, v_{*}\right) \in$ $C$ by Lemma 2.3(c). To determine $v_{*}$, notice that $v_{n+1} \rightarrow v_{*}$ by (4.5). Thus $v_{*}=g\left(v_{*}\right)$, or equivalently, $v_{*}^{2}-2 v_{*}+1=0$, so that $v_{*}=1$. Since $\sup W=\left(1, v_{*}\right)=(1,1)$, then $(1,1) \in C$ by Lemma $2.3(\mathrm{c})$. Because $(1,1)$ is a maximal element of $X$, then $(1,1)=\max C$. Moreover, $G(1,1)=(1,1)$, so that $(1,1)$ is a fixed point of $G$.

The first $m+1$ elements of the w-o chain $C$ of $a G$-iterations can be estimated by the following Maple program $($ floor $(\cdot)=[\cdot])$ :

$\mathrm{x}:=\min (1,1-$ floor $(\mathrm{u})+$ floor $(\mathrm{v})): \mathrm{y}:=\left(\right.$ floor $\left.(\mathrm{u})+\mathrm{v}^{2}\right) / 2:(\mathrm{u}, \mathrm{v}):=(1,0): \mathrm{c}[0]:=(\mathrm{u}, \mathrm{v}):$

for $\mathrm{n}$ to $\mathrm{m}$ do $(\mathrm{u}, \mathrm{v}):=(\max (\mathrm{x}, \mathrm{u})$, evalf $(\max (\mathrm{y}, \mathrm{v})) ; \mathrm{c}[\mathrm{n}]:=(\mathrm{u}, \mathrm{v})$ end do;

For instance, $\mathrm{c}[100000]=(1,0.99998)$.

The verification of the following properties are left to the reader.

(i) If $c=(u, v) \in X, u<1$, and $v<1$, then the elements of w-o chain $C$ of $a G$-iterations, after two first terms if $u<1$, are of the form $\left(1, w_{n}\right), n=0,1, \ldots$, where $\left(w_{n}\right)_{n=0}^{\infty}$ is increasing and converges to 1 . Thus $(1,1)$ is the maximum of $C$ and a fixed point of G.

(ii) If $a=(u, 1), u<1$, or $a=(1,-1)$, then $C=\{a,(1,1)\}$.

(iii) If $a=(1,0)$, then $G^{2 k} a=\left(1, z_{k}\right)$ and $G^{2 k+1} a=\left(0, y_{k}\right), k \in \mathbb{N}_{0}$, where the sequences $\left(z_{k}\right)$ and $\left(y_{k}\right)$ are bounded and increasing. The limit $z$ of $\left(z_{k}\right)$ is the smaller real root of $z^{4}-8 z+4=0 ; z \approx 0.50834742498666121699$, and the limit $y$ of $\left(y_{k}\right)$ is $y=$ $(1 / 2) z^{2} \approx 0.12920855224528457650$. Moreover $G(1, y)=(0, z)$ and $G(0, z)=(1, y)$, whence no subsequence of the iteration $\left(G^{n} a\right)$ converges to a fixed point of $G$.

(iv) For any choice of $a=(u, v) \in P \backslash\{(1,1)\}$ the iterations $G^{n} a$ and $G^{n+1} a$ are not order related when $n \geq 2$. The sequence $\left(G^{n} c\right)$ does not converge, and no subsequence of it converges to a fixed point of $G$.

(v) Denote $Y=\left\{(u, v) \in \mathbb{R}_{+}^{2}: u^{2}+v^{2} \leq 2, v>0\right\} \cup\{(1,0)\}$. The function $G$, defined by (4.1), satisfies $G[Y] \subset Y$ and is maximalizing. The maximum of the $\mathrm{w}-\mathrm{o}$ chain of $a G$-iterations with $a=(1,0)$ is $x_{*}=(1,1)$, and $x_{*}$ is a fixed point of $G$. If $x \in Y \backslash\left\{x_{*}\right\}$, then $x$ and $G(x)$ are not comparable.

The following example shows that $G$ need not to have a fixed point if either of the hypothesis of Theorem 3.1 is not valid. 
Example 4.2. Denote $a=(1, y)$ and $b=(0, z)$, where $y$ and $z$ are as in Example 4.1. Choose $X=\{a, b\}$, and let $G: X \rightarrow X$ be defined by (4.1). $G$ is maximalizing, but $G$ has no fixed points, since $G(a)=b$ and $G(b)=a$. The last hypothesis of Theorem 3.1 is not satisfied.

Denoting $c=(1, z)$, then the set $X=\{a, b, c\}$ is a complete join lattice, that is, every nonempty subset of $X$ has the supremum in $X$. Let $G: X \rightarrow X$ satisfy $G(a)=b$ and $G(b)=$ $G(c)=a$. $G$ has no fixed points, but $G$ is not maximalizing, since $G(c)<c$.

Example 4.3. The components $u=1, v=1$ of the fixed point of $G$ in Example 4.1 form also a solution of the system

$$
u=\min \{1,1-[u]+[v]\}, \quad v=\frac{[u]+v^{2}}{2} .
$$

Moreover a Maple program introduced in Example 4.1 serves a method to estimate this solution. When $m=100000$, the estimate is $u=1, v=0.99998$.

Remark 4.4. The standard "solve" and "fsolve" commands of Maple 12 do not give a solution or its approximation for the system of Example 4.3.

In Example 4.1 the mapping $G$ is nonincreasing, nonextensive, nonascending, not semiincreasing upward, and noncontinuous.

Chain $C(a)$ is compared in [10] with three other chains which generalize the sequence of ordinary iterations $\left(G^{n}(a)\right)_{n=0}^{\infty}$, and which are used to prove fixed point results for $G$. These chains are the generalized orbit $O(a)$ defined in [10] (being identical with the set $W(a)$ defined in [11]), the smallest admissible set $I(a)$ containing $a$ (cf. [12-14]), and the smallest complete $G$-chain $B(a)$ containing $a$ (cf. $[10,15])$. If $G$ is extensive, and if nonempty chains of $X$ have supremums, then $C(a)=O(a)=I(a)$, and $B(a)$ is their cofinal subchain (cf. [10, Corollary 7]). The common maximum $x_{*}$ of these four chains is a fixed point of $G$. This result implies Bourbaki's Fixed Point Theorem.

On the other hand, if the hypotheses of Theorem 3.4 hold and $x \in C(a) \backslash\left\{a, x_{*}\right\}$, then $x$ and $G(x)$ are not necessarily comparable. The successor of such an $x$ in $C(a)$ is $\sup \{x, G(x)\}$ by [14, Proposition 5]. In such a case the chains $O(a), I(a)$ and $B(a)$ attain neither $x$ nor any fixed point of $G$. For instance when $a=(0,0)$ in Example 4.1, then $C(a)=\{(0,0)\} \cup C$, where $C$ is the w-o chain of $(1,0) G$-iterations. Since $\left(G^{n}(0,0)\right)_{n=0}^{\infty}=\{(0,0)\} \cup\left(G^{n}(1,0)\right)_{n=0}^{\infty}$, then $B(a)$ does not exist, $O(a)=I(a)=\{(0,0),(1,0)\}$ (see [10]). Thus only $C(a)$ attains a fixed point of $G$ as its maximum. As shown in Example 4.1, the consecutive elements of the iteration sequence $\left(G^{n}(1,0)\right)_{n=0}^{\infty}$ are unordered, and their limits are not fixed points of $G$. Hence, in these examples also finite combinations of chains $W\left(a_{i}\right)$ used in [16, Theorem 4.2] to prove a fixed point result are insufficient to attain a fixed point of $G$.

Neither the above-mentioned four chains nor their duals are available to find fixed points of $G$ if $a$ and $G(a)$ are unordered. For instance, they cannot be applied to prove Theorems 3.1 and 3.5 or Propositions 3.2 and 3.7.

\section{References}

[1] N. Bourbaki, Eléments de Mathématique, I. Théorie des Ensembles, Fascicule de Résultats, Actualités Scientifiques et Industrielles, no. 846, Hermann, Paris, France, 1939.

[2] W. A. Kirk, Fixed Point Theory: A Brief Survey, Notas de Matematicas, no. 108, Universidas de Los Andes, Mérida, Venezuela, 1990. 
[3] J. Klimeš, "A characterization of inductive posets," Archivum Mathematicum, vol. 21, no. 1, pp. 39-42, 1985.

[4] S. Heikkilä, "Fixed point results for semi-increasing mappings," to appear in Nonlinear Studies.

[5] S. Heikkilä, "Monotone methods with applications to nonlinear analysis," in Proceedings of the 1st World Congress of Nonlinear Analysts, vol. 1, pp. 2147-2158, Walter de Gruyter, Tampa, Fla, USA, 1996.

[6] S. Heikkilä, "A method to solve discontinuous boundary value problems," Nonlinear Analysis, vol. 47, pp. 2387-2394, 2001.

[7] S. Heikkilä, "On recursions, iterations and well-orderings," Nonlinear Times and Digest, vol. 2, no. 1, pp. 117-123, 1995.

[8] S. Carl and S. Heikkilä, Nonlinear Differential Equations in Ordered Spaces, Chapman \& Hall/CRC, Boca Raton, Fla, USA, 2000.

[9] S. Heikkilä and V. Lakshmikantham, Monotone Iterative Techniques for Discontinuous Nonlinear Differential Equations, vol. 181 of Monographs and Textbooks in Pure and Applied Mathematics, Marcel Dekker, New York, NY, USA, 1994.

[10] R. Manka, "On generalized methods of successive approximations," to appear in Nonlinear Analysis.

[11] S. Abian and A. B. Brown, "A theorem on partially ordered sets, with applications to fixed point theorems," Canadian Journal of Mathematics, vol. 13, pp. 78-82, 1961.

[12] T. Büber and W. A. Kirk, "A constructive proof of a fixed point theorem of Soardi," Mathematica Japonica, vol. 41, no. 2, pp. 233-237, 1995.

[13] T. Büber and W. A. Kirk, "Constructive aspects of fixed point theory for nonexpansive mappings," in Proceedings of the 1st World Congress of Nonlinear Analysts, vol. 1, pp. 2115-2125, Walter de Gruyter, Tampa, Fla, USA, 1996.

[14] S. Heikkilä, "On chain methods used in fixed point theory," Nonlinear Studies, vol. 6, no. 2, pp. 171$180,1999$.

[15] B. Fuchssteiner, "Iterations and fixpoints," Pacific Journal of Mathematics, vol. 68, no. 1, pp. 73-80, 1977.

[16] K. Baclawski and A. Björner, "Fixed points in partially ordered sets," Advances in Mathematics, vol. 31, no. 3, pp. 263-287, 1979. 